\title{
S42. Affective disorders: biological and social aspects
}

THE EFFECT OF LITHIUM TREATMENT ON SURVIVAL OF PATIENTS WITH AFFECTIVE DISORDERS

B Ahrens, M Alda, E Grof, P Grof, G Lenz, B Müller-Oerlinghausen, $M$ Schou, C Simhandl, $K$ Thau, $P$ Vestergaard, $R$ Wolf, $T$ Wolf

Department of Psychiatry, Freie Universität Berlin, Eschenallee 3, 14050 Berlin, Germany; and Departments of Psychiatry: University of Ottawa, Canada; University of Vienna, Vienna, Austria; University of Aarhus, Aarhus, Denmark

Death is the most severe complication of affective disorders. The overall mortality rate of these patients extends up to three times in excess of the general population; if not adequately treated, $10 \%$ to $20 \%$ end their own lives. The excess mortality was observed before antidepressants were widely introduced and persisted after they had been in use for many years. The question thus arises as to whether psychiatric treatment can reduce the excess mortality in affective disorders.

Recently, studies have indicated that longterm lithium treatment can, in principle, reduce the expected suicidal activity and overall mortality of such patients. In a study by IGSLi (International Group for the Study of Lithium-treated patients), we observed that the mortality of manicdepressive and schizoaffective patients given longterm lithium treatment was reduced to a level that was indistinguishable from that of the general population. However, the mortality-reducing property of lithium can be strengthened or weakened by different moderating variables such as the length of treatment, treatment setting and compliance.

Data of an IGSLi study, carried out in Lithium Clinics in Austria, Canada, Denmark and Germany, concerning the impact of longterm lithium treatment on the mortality of 1,000 patients with manic depressive and schizoaffective illness, will be presented. A minimum length of two years of lithium treatment is needed to achieve a significant reduction of the excess mortality of manic-depressive patients.
EARLY RESPONSE AS A PROGNOSTIC CRITERIA OF FLUVOXAMINE EFFICACY IN MANAGEMENT OF DEPRESSION

\section{T. Serebriakova}

National Research Centre for Social and Forensic Psychiatry, Kropotinsky per. 23, 119839, Moscow, Russia.

Antidepressants $(A D)$ are the drug of choice for management of depression, however the $A D$ benefits are not equal in all cases, so, the early prediction of $A D$ efficacy is of particular importance. The study was designed to evaluate the predictive potency of the early response to the first oral dose of Fevarin (FEV) in comparative study with Tryptizol (TRY) and Tolvon (TOL).

90 adult outpatients with mean age $=39,0$ meeting the DSM-III criteria for major depressive disorder entered the 6-week flexible-dose study preceded by 1 -week washout period and 24-hours evaluation period after the first ora dose of $A D$ ( $F E V=50 \mathrm{mg}, T A Y=25 \mathrm{mg}, T O L=30 \mathrm{mg}$ ). Patients were randomly assigned to one of the three treatment group with 30 patients in each. The assessments of the early response consisted of Kelliner Symptom-Rating Test and an originally designed self-rating scale to measure the subjective estimate of symptoms' changers and the attitude to the medication. Efficacy evaluations were conducted on the last day of the washout period and weekly thereafter, and included HAM-D, SDS Index, CGI. The results were analyzed with the application of t-Test and Wilcoxon Test. The 4 types of early response were distinguished according to the assessment results of the 24-hours evaluating period. The investigation of the relationship between the type of early response and clinical outcome showed significant correlation for FEV, less significant for TOL and no correlations for TRY. The results suggest that the predictive potency of the early response to the $A D$ treatment is connected with the selective effects on serotonin system. 
ETHNICITY, SEASON AND MOOD DISORDER PRESENTATION: IS THERE A RELATIONSHIP? A PRELIMINARY SOUTH AFRICAN STUDY

C.P. Szabo, M.J. Terreblanche, G.A.D. Hart

Department of Psychiatry, University of the Witwaterstand Medical School 7 York Road, Parktown 2193, South Africa

Statistically significant seasonal pattems for Mood disorder presentation have recently been documented in South African patient samples, one was African $(p=0.0007)$, the other Caucasian $(p=0.0011)$. The present study attempted to establish whether ethnicity plays a role in Mood disorde presentation as a function of season. The earlier studies were retrospective ones reviewing admission data over a one year period at two separate locations within a specific geographical area. The two data sets were compared looking a seasonal patterns of presentation for both Mood and non-mood disorder patients. A statistically significant difference was wound for Mood disorder $(p=0.034)$, but not non-mood disorder presentations $(p=0.964)$. In addition, differences were found pertaining to Mood disorder presentations as a percentage of total presentations (African $=20 \%$; Caucasian $=40 \%$ ) as well as the nature of presenting Mood disorders (African: $60 \%=$ Mania; Caucasian: $77 \%=$ Depression). The results are preliminary and not without limitations, but suggest that further investigation es warranted given that these differences may have important management implications
LINKAGE ANALYSES BETWEEN MANIC-DEPRESSIVE ILLNESS AND SELECTED MARKERS

H Ewald', O Mors ${ }^{1}$, T Flint ${ }^{2}$, H Eiberg ${ }^{3}$ \& \&A Kruse ${ }^{2}$

${ }^{1}$ Institute of Basic Psychiatric Research, Department of Psychiatric Demography and Department of Biological Psychiatry, Psychiatric Hospital in Aarhus, ${ }^{2}$ Institute of Human Genetics, Aarhus University, ${ }^{3}$ University Institute of Medical Genetics, Panum, Copenhagen, Denmark.

As participants in the continuing search for major genes involved in the etiology of manic-depressive illness, we have at the present time tested approximately 85 markers.

The material consists of two large Danish families, in which several members have manic-depressive illness. ICD-10 diagnoses based on interview with patients and relatives, case records from hospitals and general practitioners are used.

Linkage analyses using the lod score method and sib-pair methods have been applied. Models with dominant as well as recessive mode of inheritance have been investigated. Conservative genetic parameters, affecteds-only analyses and several other measures for minimizing misclassification are used.

Apart from a random, as well as a more systematic search, several candidate genes and regions have been investigated, including genes encoding neurotransmitter enzymes and neurotransmitters, and the long arm of chromosome 11.

Results from selected markers will be presented.

An association study of bipolar affective disorder using DNA markers

H Yoneda, T Sakai, Y Inayama, T Ishida, Y Nonomura, Y Kono, J Koh, K Kuroda, H Higashi and H Asaba.

Department of Neuropsychiatry, Osaka Medical College,

Daigaku-cho 2-7, Takatsuki, Osaka, 569, Japan.

Family, twin and adoption studies have shown that genetic factors play an important role in the etiology of affective disorders. After introducing positional cloning to psychiatric genetics, linkage and association studies using DNA markers have been conducted to identify the responsible genes for affective disorders. However the results of these studies remain inconclusive. We conducted an association study between bipolar affective disorder and Tyrosine Hydroxylase (TH), adrenaline receptor (ADR), serotonin receptor (5HTR) and pseudoautosomal DNA markers in biologically unrelated bipolar patients and normal controls who are native Japanese living in the western area of Japan. Diagnosis was made according to DSM-III-R criteria. DNA samples digested by restriction enzymes were hybridized with DNA markers; DXYS17, 20, 28 and $\mathrm{MIC} 2$ on pseudoautosomal region (Xp22.32; Yp11.3), TH (11p15.5), alpha 2 ADR(10p24-26), beta 2 ADR (5q32-34). 5HT1 a (5cen-q11) and 5HT2 (13q14-21) receptor genes were amplified by $\mathrm{PCR}$. PCR products were digested with restriction enzymes. DXYS17, 28, MIC2, TH, 5HTR1a, 5HTR2, alpha2 ADR, beta2 ADR showed no significant association with bipolar affective disorder. However DXYS20 showed significantly higher frequencies of the patients with the EcoRI A4 allele or Taql A3 allele compared with normal controls. The positive association between DXYS 20 and bipolar affective disorder indicates that the pseudoautosomal region may play an important role in developing bipolar affective disorder. 
A MULTICENTER DOUBLE BLIND TRIAL OF PAROXETINE VS. AMITRIPTYLINE IN DEPRESSED IN-PATIENTS

CH Stuppaeck, C. Geretsegger, AB Whitworth, H Schubert, T Platz, P Koenig, H Hinterhuber, WW Fleisch-

hacker

Dept. of Psychiatry, Innsbruck University Clinics, Anichstrasse 35, A-6020 Innsbruck, Austria

The phenylpiperidine derivative paroxetine is a selective serotonin reuptake inhibitor. In a doubleblind six week trial paroxetine was compared to amitriptyline in hospitalized patients suffering from major depression (DSM III). 153 patients were enrolled in the study in seven centers in Austria and Germany. Results showed similar efficacy of

both drugs after six weeks. The differences between groups in MADRS and CGI ratings did not reach statistical significance at any time point.

side effects were distributed similarly but with a significantly higher incidence of anticholinergic effects in patients treated with amitriptyline

( $p<0.001)$, whilst agitation and insomnia were registered more often in the paroxetine group.

This study supports the antidepressive efficacy of paroxetine in a sample of severely depressed inpatients. 